\title{
REVIEW
}

\section{Spectral selectivity in infrared thermal detection}

\author{
Joseph J Talghader, Anand S Gawarikar and Ryan P Shea
}

A review is made of the physics and technology of spectrally selective thermal detectors, especially those operating at non-cryogenic temperatures. The background radiation noise fluctuations are rederived for arbitrary spectral characteristics. Infrared absorption due to phonons and free carriers is discussed followed by a review of published works on artificial infrared absorption materials such as patterned grids, nanoparticles, plasmonic structures, metamaterials and others. Subsequently, the literature of the spectral characteristics of broadband thermal detectors and spectrally selective thermal detectors is reviewed. Finally, the authors speculate on the directions that future research and development in the area will take regarding architectures, sensitivity and spectral characteristics.

Light: Science \& Applications (2012) 1, e24; doi:10.1038/lsa.2012.24; published online 31 August 2012

Keywords: infrared detectors; infrared materials; radiation noise; thermal detectors

\section{INTRODUCTION}

The physics of nanomechanical structures has attracted great interest in the past two decades with studies of their phonons, ${ }^{1,2}$ heat conduction $^{3}$ and vibrational states, ${ }^{4,5}$ among other topics. In the past several years, this work has been complemented by research into coherent thermal emission devices. ${ }^{6-8}$ Such advances in nanostructures and infrared sources seem to have positioned scientists and engineers for a new look at the old technology of the infrared thermal detector, which requires an extremely small thermal mass to interact with one or more electromagnetic modes. Traditional thermal detectors, such as microbolometers, have been considered as broadband devices that image spectrally uniform objects, but there are many applications where spectral selectivity would be critical, such as chemical detection and target recognition, not to mention fundamental studies of cavity quantum electrodynamics. In this review, we survey the current stateof-the-art for spectrally selective infrared thermal detectors. We begin with a rederivation of the radiation noise of thermal detectors but modified for wavelength-dependent emissivity. We then discuss infrared absorption mechanisms in common solids and current artificial materials. In the section on 'Spectral properties of infrared materials: bulk', we discuss the spectral properties of current imaging thermal detectors, and then discuss the characteristics of narrowband or multicolor detectors. Finally, we speculate on some ways that the field will progress in the coming decade.

\section{RADIATION NOISE}

Most of the theory for thermal detectors was developed many decades ago in the years following World War II. ${ }^{9,10}$ While the authors of these treatments realized that thermal detectors could have spectral variations, almost all of the thermal detectors of that period were designed to absorb uniformly across wide spectral bands. ${ }^{11,12}$ This is quite different from the progression in the technology of microwave absorbers, where frequency-dependent absorption was studied thoroughly at around the same time. ${ }^{13-17}$ For thermal detectors, it was natural then that the special case of uniform absorption was used when noise calculations were made and performances compared to that of photodetectors. Indeed, prior to the last decade, only a relatively small number of works on thermal detectors covered spectral selectivity, and few, if any, current archival works describe how interference structures alter background-limited noise. To address this, we will extend the treatments of Jones, ${ }^{9}$ Lewis ${ }^{10}$ and Fellgett ${ }^{18}$ to cover spectrally selective devices.

The background or radiation noise of a thermal detector originates from fluctuations in the total energy of the photons being emitted or absorbed from the detector structure. In contrast, the background noise of a photodetector originates from fluctuations in the number of photons emitted or absorbed. So, while the derivation below requires the number fluctuations as an introductory quantity, it is not our main interest, per se. With that in mind, fundamental thermodynamics ${ }^{19}$ tells us that the mean square fluctuation in the number of particles in a single mode of a Bose-Einstein gas fluctuates according to:

$$
\overline{\Delta n^{2}}=\bar{n}+\bar{n}^{2}
$$

where $\bar{n}$ is the average number of particles (photons) in the mode. The mean square energy fluctuations for the system for a specific spectral region can be obtained by multiplying by the square of the energy of a photon:

$$
\overline{\Delta E^{2}}{ }_{v}=N(h v)^{2} \overline{\Delta n^{2}}
$$

where $h$ is Planck's constant, $v$ is the photon frequency and $N$ is the mode density in the spectral region of interest. In Bose-Einstein statistics, the average number of particles (photons) per state is: 


$$
\bar{n}=\frac{1}{e^{\frac{h v}{k_{B} T}}-1}
$$

where $k_{B}$ is Boltzmann's constant and $T$ is the temperature.

In order to calculate $\overline{\Delta n^{2}}$ and $\overline{\Delta E^{2}}{ }_{v}$, we still need to know $N$, the density of states in the spectral region of interest. This is most easily done in $k$-space, where a spherical shell of thickness $\mathrm{d} k$ will have a volume of $4 \pi k^{2} \mathrm{~d} k$, where $k=2 \pi v / c$. Each state of the system is an electromagnetic resonant mode of the volume in which the detector lies. In $k$-space, each mode resonance occurs at a point, $m \pi / L$, where $m$ is an integer and $L$ is a characteristic length for the volume, so we must divide $4 \pi k^{2} \mathrm{~d} k$ by $(\pi / L)^{3}$ to get the number of modes. We must also multiply by a factor of 2 to account for the two independent electromagnetic polarizations and divide by 8 , since only one octant of the spherical shell is independent. The result of this calculation is the number of states per unit volume in the frequency range of interest:

$$
N=\frac{8 \pi v^{2} \mathrm{~d} v}{c^{3}}
$$

Plugging Equation (3) into (1), we obtain,

$$
\overline{\Delta n^{2}}=\frac{1}{e^{\frac{h v}{k_{B} T}}-1}+\left(\frac{1}{e^{\frac{h v}{k_{B} T}}-1}\right)^{2}=\frac{e^{\frac{h v}{k_{B} T}}}{\left(e^{\frac{h v}{k_{B} T}}-1\right)^{2}}
$$

and then substituting Equations (4) and (5) into Equation (2), we obtain the energy fluctuations of a thermal detector within a specific spectral range:

$$
\overline{\Delta E^{2}}{ }_{v}=\frac{8 \pi v^{2} \mathrm{~d} v}{c^{3}}(h v)^{2} \frac{e^{\frac{h v}{k_{B} T}}}{\left(e^{\frac{h v}{k_{B} T}}-1\right)^{2}}=\frac{8 \pi h^{2} v^{4}}{c^{3}} \frac{e^{\frac{h v}{k_{B} T}} \mathrm{~d} v}{\left(e^{\frac{h v}{k_{B} T}}-1\right)^{2}}
$$

and with this we can obtain the total energy fluctuations of a blackbody by integrating across all regions of the spectrum (the details of this integration are described in Refs. 10 and 13):

$$
\overline{\Delta E^{2}}=\int_{0}^{\infty} \overline{\Delta E^{2}}{ }_{v}=\int_{0}^{\infty} \frac{8 \pi h^{2} v^{4}}{c^{3}} \frac{e^{\frac{h v}{k_{B} T}} \mathrm{~d} v}{\left(e^{\frac{h v}{k_{B} T}}-1\right)^{2}}=\frac{16 k_{B} \sigma T^{5}}{c}
$$

where $\sigma$ is the Stefan-Boltzmann constant, $\sigma=5.67 \times$ $10^{-8} \mathrm{~W} \mathrm{~cm}^{-2} \mathrm{~K}^{-4}$.

For most imaging thermal detectors, which operate at room temperature and absorb efficiently across the $8-14 \mu \mathrm{m}$ range, the blackbody derivation is a good metric against which to compare noise. However, for spectrally selective thermal detectors, we need to modify the above expression by the emissivity, $\varepsilon(v, \theta, \phi)$, since only a fraction of the photons incident on the detector actually get absorbed (with an equivalent contribution from emission). This changes the above integration to:

$\overline{\Delta E^{2}}=\int_{0}^{\infty} \overline{\Delta E^{2}}{ }_{v}=\int_{v_{1}}^{v_{2}} \int_{0}^{\pi} \int_{0}^{2 \pi} \frac{2 h^{2} v^{4}}{c^{3}} \frac{e^{\frac{h v}{k_{B} T}}}{\left(e^{\frac{h v}{k_{B} T}}-1\right)^{2}} \varepsilon(v, \theta, \phi) \sin \theta \mathrm{d} \phi \mathrm{d} \theta \mathrm{d} v$

In this expression, we have also taken out $4 \pi$ steradians so that an integration of the emission as a function of solid angle can be made. Note also that this integral must be applied twice: once at the temperature of the detector for emission processes and then separately at the temperature of the background for absorption processes.
A material that only absorbs in a small spectral region will have significantly lower background noise than one that absorbs uniformly across the infrared, because fewer photons are absorbed or emitted from the material. This arises from the reduced thermal conductance, which has been measured in recent thin film devices. ${ }^{20}$ The effects of photon emission and absorption on the energy fluctuations are the same by Kirchoff's Law, and the similarity applies for light of any particular wavelength range, polarization or direction.

There are a number of ways that spectral selectivity can be achieved. Perhaps the most obvious and trivial way is to take a standard thermal detector, such as a microbolometer, and put a Fabry-Perot filter in front of it. This blocks the portions of the spectrum outside of the desired spectral region, but it does little to change the background noise of the detector. The reason is that the detector itself is still a nearblackbody that is emitting and absorbing photons in all directions. The Fabry-Perot filter will act as a mirror in its own direction, which will locally reduce the emissivity of the background, but this is only a small fraction of the solid angle and spectrum surrounding the detector. In cryogenic systems, one can place the detector in a cooled shield and then additionally cool the detector and the filter. This process reduces the noise dramatically, to a level where only the background in the spectral region and direction of the filter makes a contribution. Unfortunately, cryogenic cooling is extremely unwieldy and expensive, so in most practical thermal imaging and detection systems, the devices remain uncooled.

Spectrally selective systems can do better than simple filtering, and at the risk of being somewhat simplistic, we will split our treatment into two general groups: those that obtain spectral selectivity using materials and those that use interference structures. In reality, of course there is significant overlap between the two groups when materials and interference are used in tandem to achieve a certain set of spectral characteristics. We have also taken the liberty of lumping a number of devices using cavities, antennas, gratings, photonic crystals, metamaterials, plasmonic structures, and others all into the interference category. Before delving into the literature, we will examine the ideal case for both groups.

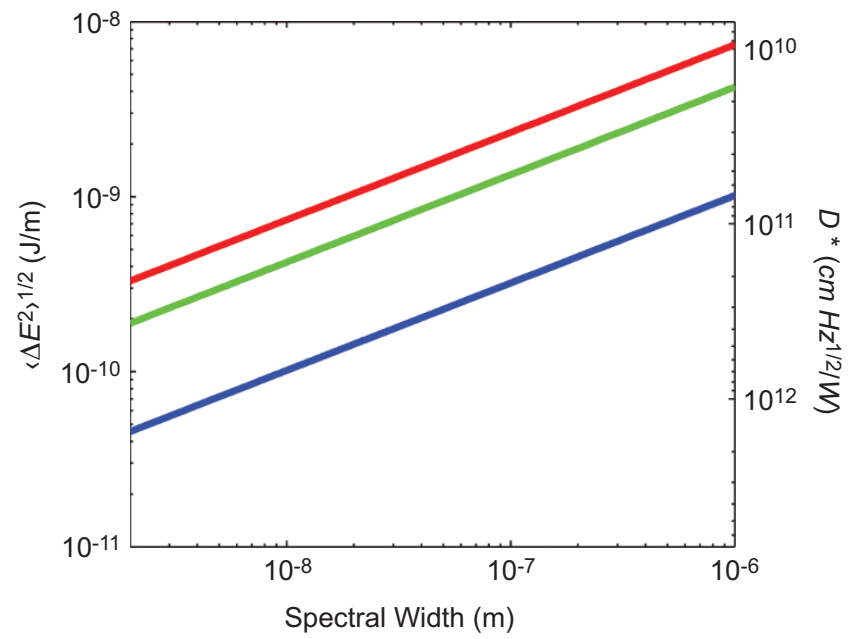

Figure 1 RMS energy fluctuations (left axis) and detectivity (right axis) for a material that absorbs perfectly within a small wavelength range centered at $\lambda=10 \mu \mathrm{m}$ but has zero absorption outside that range. The integration was taken over a hemisphere rather than the full sphere of Equation (8), and the detector thermal emission was assumed to be Lambertian. The background was taken as a perfect blackbody at $290 \mathrm{~K}$ (blue), $800 \mathrm{~K}$ (green) and $1300 \mathrm{~K}$ (red). The detector temperature was assumed to be $290 \mathrm{~K}$. RMS, root mean square. 
a

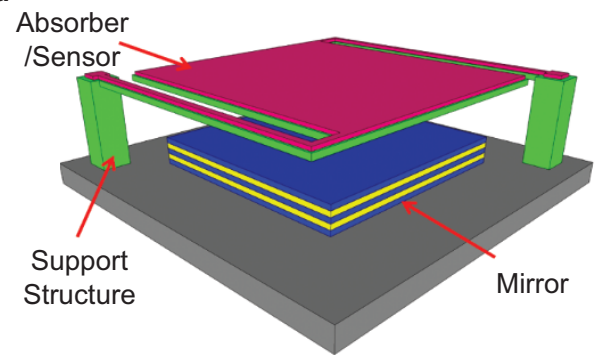

b

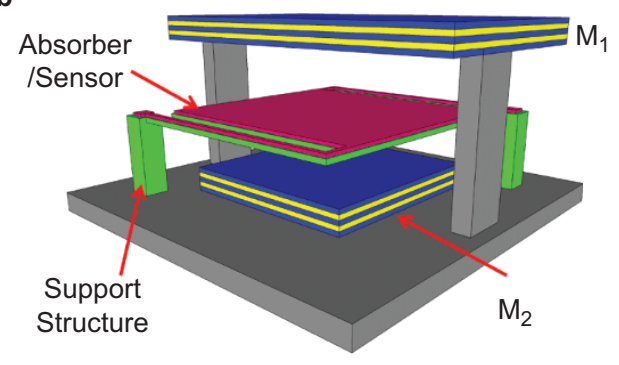

Figure 2 Diagram of cavity coupled (a) and Fabry-Perot couple (b) detector configurations.

A perfect spectrally selective system might be one where the material of the detector absorbs all light between two frequencies but is transparent to all other frequencies. In other words, $\varepsilon=1$ for $v_{1}<v<v_{2}$ and $\varepsilon=0$ for $v<v_{1}$ and $v>v_{2}$. Figure 1 shows the noise fluctuations for such a system. Here, we have a spectral absorption band that is centered at $\lambda=10 \mu \mathrm{m}$ and varies in width on a logarithmic scale. It is interesting to see that while there is a large increase in the noise fluctuation energy from 290 to $800 \mathrm{~K}$, there is a much smaller increase from 800 to $1300 \mathrm{~K}$. The explanation is that $\lambda=10 \mu \mathrm{m}$ is approximately at the peak of the thermal blackbody radiation distribution at $290 \mathrm{~K}$ and in that temperature range, power is added very efficiently to that spectral region. Much of the $P \approx \sigma \varepsilon T^{4}$ power of the Stefan-Boltzmann Law is added there. However, as the temperature increases, the peak of the distribution shifts to shorter wavelengths and less power is allotted to the $10-\mu \mathrm{m}$ range. So, even though the overall radiation energy jumps tremendously between 800 and $1300 \mathrm{~K}$, the energy in the spectral range of the detector levels off.

Another way to achieve spectral selectivity is to couple a low absorption material with an interference structure. There are a variety of interference structures, but the concept of adding spectral components in phase to enhance a desired spectral region, direction or polarization while inhibiting others via destructive interference is common to all. We should also explicitly note that spectral selectivity implies some degree of spectral coherence. This has been widely disseminated in publications on coherent thermal emission ${ }^{21,22}$ and has been used in photodetectors $^{23}$ and thermal detectors ${ }^{24}$ for many years but recently has attracted wide press for its application as an antilaser. ${ }^{25}$

A Fabry-Perot optical cavity is perhaps the most archetypical interference structure, and a coupled-absorption cavity and detector is shown in Figure $2 \mathrm{a}$ and $2 \mathrm{~b}$. There are several ways to perform the cavity coupling, but one of the most straightforward is to modify a Gires-Tournois cavity, ${ }^{26}$ that is, a lossless Fabry-Perot cavity with a $100 \%$ reflecting back mirror. This type of cavity is frequently used when a change in spectral phase (dispersion) is desired without a change in spectral amplitude. However, in this case, a thin absorbing layer or structure is placed, either as one of the mirrors or within the central cavity. If the absorption of this layer is carefully chosen, then at one wavelength and in one direction, all incident light is coupled into the layer. The remaining light that is near the cavity optical axis is reflected away by the mirrors. Light from other directions is absorbed by the detector plate at its relatively low single-pass absorption value. Therefore, the system accepts the entire signal from the desired direction and spectral band but relatively little of the unwanted background. In the limit as the width of the spectral resonance decreases towards zero, the background fluctuation noise approaches within a small factor of that of a cold-shielded and cold-filtered system, even without any cooling. This explanation is something of a simplification, since there will be harmonics of the main resonance, non-uniformity in the mirror reflectivity, and multiple reflections or imperfect coupling of some of the off-axis light. However, these do not alter the basic concept. We should also point out that this is merely a one-dimensional cavity. Theoretically, better cavity coupling could be achieved by confining light in two or three dimensions using photonic crystal techniques; ${ }^{22,27}$ indeed, the detector itself could be patterned as part of the photonic structure; however, the concomitant difficulties of fabricating a compatible thermal isolation structure have apparently precluded this approach from being experimentally implemented to date.

Figure 3 shows the energy fluctuations for an ideal Fabry-Perot cavity with uniform absorption between the two mirrors normalized to those of the perfect absorber from Figure 1. The cavity emissivity has been integrated over a hemisphere and takes the form:

$$
\varepsilon_{\text {cavity }}=1-|r|^{2}=1-\frac{\left(\sqrt{R_{1}}-e^{-\alpha l}\right)^{2}+4 \sqrt{R_{1}} e^{-\alpha l} \sin ^{2} \phi}{\left(1-\sqrt{R_{1}} e^{-\alpha l}\right)^{2}+4 \sqrt{R_{1}} e^{-\alpha l} \sin ^{2} \phi}
$$

where $R_{1}$ is the reflectivity of the top mirror, $\alpha$ is the absorption coefficient of the central structure, $l$ is the distance between the two mirrors and $\phi$ is the phase accumulated in one traversal of the cavity.

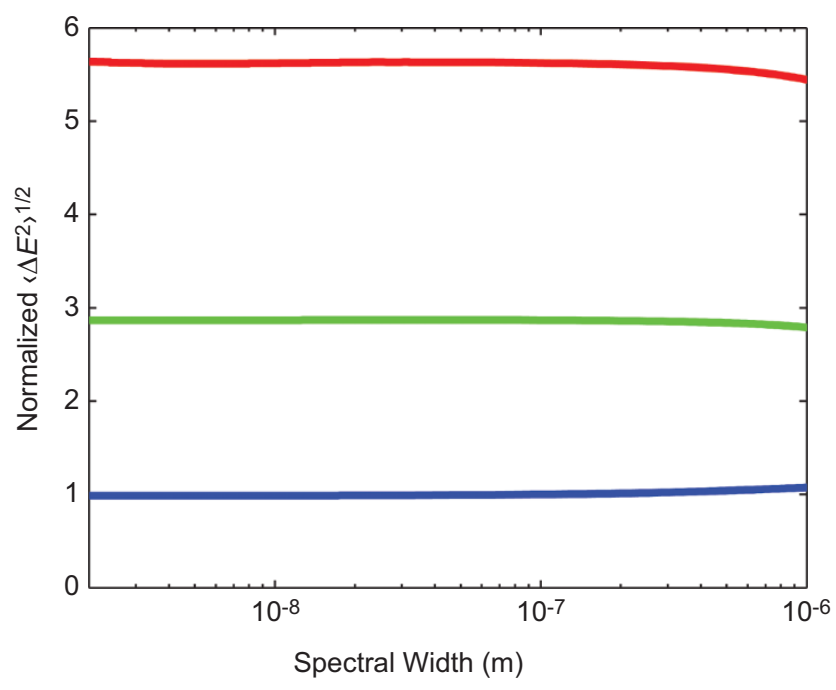

Figure 3 Mean square energy fluctuation for an ideal cavity with central absorber integrated over a hemisphere (the second hemisphere is ignored because of the perfect back mirror) normalized to the perfect absorber from Figure 1. The spectral width describes a resonance covering a small wavelength range centered at $\lambda=10 \mu \mathrm{m}$. Three background temperatures are shown: $290 \mathrm{~K}$ (blue), $800 \mathrm{~K}$ (green) and $1300 \mathrm{~K}$ (red). The detector temperature is $290 \mathrm{~K}$. 
We can see that the fluctuations for the cavity are higher than that for the more ideal case when the background temperature is high. But for a 290-K background, the fluctuations for the Fabry-Perot cavity are equal to that of the ideal case. In practice, instead of a cavity with mirrors of infinite extent, a practical cavity would only impact light incident from a certain range of solid angles. Light incident from outside this cone would miss the cavity but still strike the detector plate. We can modify our treatment of the noise fluctuations to handle this case, but we find that there is very little difference from the infinite cavity, a difference of only approximately $5 \%$, where the case of a finite cavity is slightly better than that of infinite cavity.

\section{SPECTRAL PROPERTIES OF INFRARED MATERIALS: BULK}

There are a large number of infrared absorption mechanisms in bulk solids, and, in theory, any could be used in an absorption layer for a thermal detector. Some of these, such as bandgap absorption and impurity absorption, are mechanisms that are commonly seen for shorter wavelength light in the visible and near-infrared and may be more widely known to many readers; therefore, we will confine our short discussion of bulk absorption mechanisms to phonon absorption and free carrier absorption, which along with impurity and bandgap absorption, dominate the infrared spectra of most background materials.

Perhaps the most basic infrared absorption is that of a photon by a molecular bond to create a quantum of energy in one or more vibrational states. This absorption is strongest when the bond has substantial ionic character, that is, there is a significant charge distribution across the molecule, so that the vibration of the molecule creates a resonant electromagnetic field at one or more vibrational mode frequencies. Mathematically, the generated field can be expanded, and the first and strongest term represents a dipole transition. ${ }^{28}$ In a crystalline solid, these quanta of vibrational energy take the form of optical phonons, which is a quantum of energy in one of the lattice vibrational modes, in particular, a mode where the atoms of the primitive basis are not moving in unison. ${ }^{29-31}$ They occur in all crystalline solids that have two or more atoms in the basis of their crystal structure, but strong photon absorption can only occur when the oscillation changes the net charge distribution or dipole moment of the solid. We can further state that photons are only absorbed by optical phonons that vibrate transverse to the propagation direction (vector) of the incident field (TO phonons). However, the longitudinal optical (LO) phonons do play a significant role in the optical properties of a solid, since a very sharp drop in the absorption coefficient occurs around the longitudinal resonant frequency. This is a phenomenon explained by the Lyddane-Sachs-Teller relationship. ${ }^{29,32}$ The phonon absorption region of the infrared spectrum that lies near or between the resonant frequencies of the LO and TO phonons is known as the restrahlen region. Restrahlen absorption is very strong with absorption coefficients on the order of $10^{4}-10^{5} \mathrm{~cm}^{-1}$.

Not all molecular vibrations can interact with a single photon. Diatomic molecules, such as $\mathrm{N}_{2}$ and $\mathrm{O}_{2}$, have no electric dipole moment; therefore, their transition matrix elements are zero in the first term of the field expansion, and they absorb only weakly. Higher order terms do allow weak transitions in these molecules. For example, nitrogen has a quadrupole absorption at its fundamental vibration frequency of $2329.9 \mathrm{~cm}^{-1} \cdot 33$ It also has a collision-induced absorption where the interaction of two nitrogen molecules briefly induces a dipole which can then absorb a photon. This later process is germane to our discussion, because covalent semiconductors, such as silicon and germanium, do not have a dipole moment for their fundamental basis vibration and thus absorb light only weakly via multiple phonon processes. ${ }^{31}$ Multiphonon absorption occurs when one phonon induces a temporary dipole moment in the solid by shifting the relative positions of the atoms of the basis, which a second phonon can then cause to oscillate. This creates an interesting analogy to the collisional process of nitrogen. There are also magnetic dipole transitions such as those seen in diatomic oxygen, whose magnetic dipole originates from an unpaired electron spin. ${ }^{33}$ Higher-order processes such as these are relatively weak and seen only when a large quantity of a material is being observed.

Unfortunately, it is not as easy to use the restrahlen and other phonon absorption regions to identify solids as it is to use infrared vibrational signatures to identify molecules. One reason is the breadth of most phonon lines. The scattering time for phonons in solids is very small at room temperature, on the order of a picosecond. This scattering rapidly dephases the phonon wavefunction, which broadens the spectral lines. Thus, the phonon features of many materials overlap and cannot be distinguished. Another reason is the extreme variation in purity and crystal structure between different solids. Technically, the term phonon only applies to periodic solids, but even loosening the definition, a vibrational mode of a crystalline material will shift between a perfect crystal, a nanocrystal and an amorphous solid. Many common substances, such as rock, paint, or vegetation, appear relatively featureless in the infrared and can often be approximated with a uniform high emissivity. These materials are mixtures with many overlapping bond vibrations.

Free carrier absorption originates from the interaction of carriers with light of frequency above that of the plasma resonance. At optical frequencies, we normally associate the plasma frequency as a feature of metals, but the only critical differences between (ideal) semiconductors and metals are the use of an effective mass and some additional contributions to the dielectric constant. Of course, the carrier density in semiconductors is much lower than in metals, and this shifts the plasma resonance deep into the infrared.

An ideal classical treatment of the absorption ${ }^{32}$ leads to a $\alpha \propto N \lambda^{2}$ dependence, where $\alpha$ is the absorption coefficient, $N$ is the carrier concentration and $\lambda$ is the wavelength. However, experimental data indicates the exponent is somewhat larger than this, between 2 and 3 . Free carrier absorption is a three particle process that must involve the photon, the carrier (either an electron or hole), and a phonon or some other scattering mechanism to conserve momentum.

One of the most interesting features of free carrier absorption is its dependence on doping, which is a parameter that a designer can control. By tailoring the doping, one can create a material with arbitrarily low or high absorption. This is particularly important if one desires a low absorption for a detector material, for example, if one wishes to couple a lightly absorbing detector to a high finesse optical cavity to achieve strong absorption at a single wavelength. The wavelength dependence of the absorption could be a problem in a broadband detector, but if the desired spectral range is narrow enough, it poses no difficulties. As an example, as a traditional thermal detector material, germanium is often considered to be either transparent (undoped) or metallic (heavily doped) in the thermal infrared. However, the free carrier properties of germanium and other semiconductors have been known for many years with extensive experimental data showing intermediate values. ${ }^{34-38}$

\section{SPECTRAL PROPERTIES OF INFRARED MATERIALS: ARTIFICIAL}

The emission of thermal radiation from a heated cavity is given by Planck's law. However, if the dimensions of the cavity are comparable 

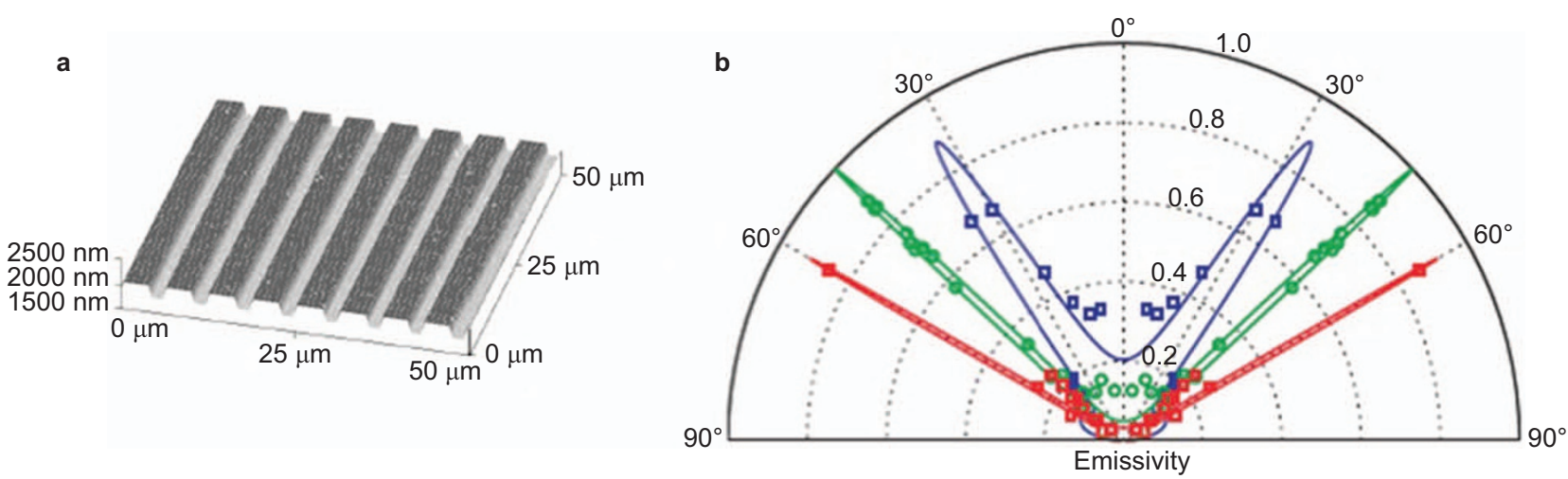

Figure 4 (a) AFM image of a SiC grating. (b) Spatial distribution of emissivity for three different wavelengths from Ref. 6. AFM, atomic force microscope.
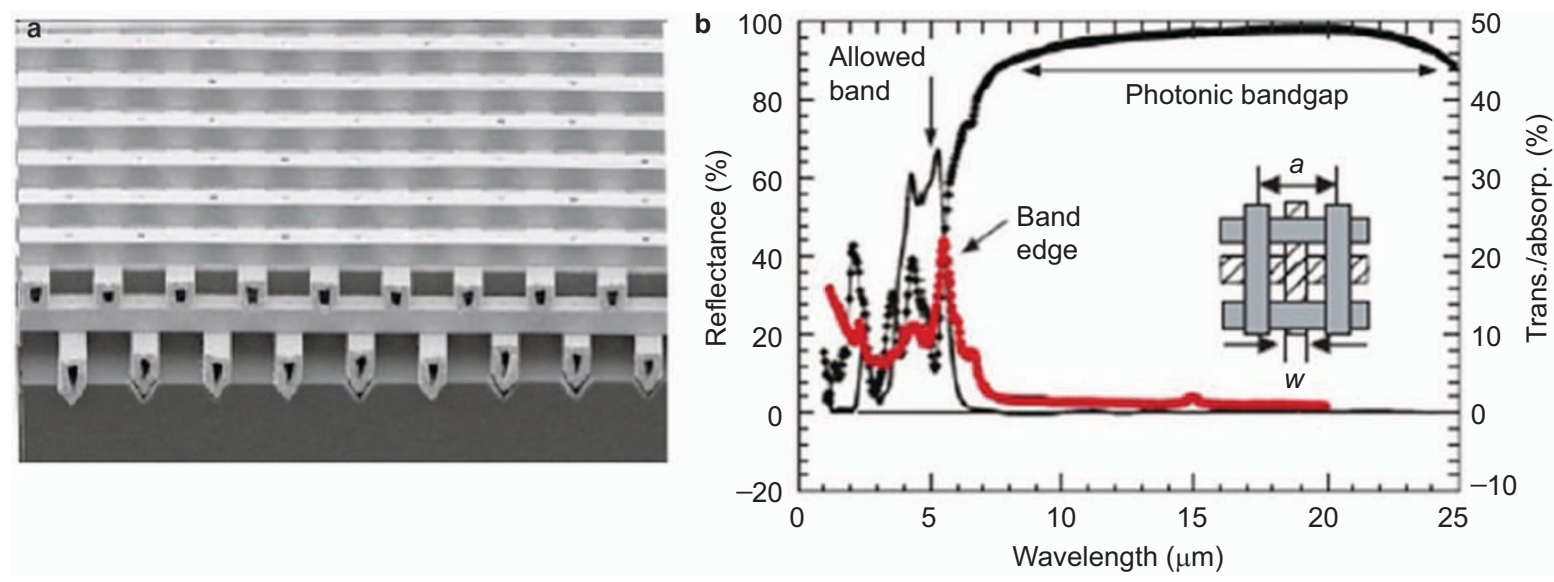

Figure 5 (a) SEM image of three-dimensional photonic crystal made from tungsten. (b) Reflectance (black) and absorption (red) spectra. ${ }^{27}$ SEM, scanning electron microscope.

to the wavelength, $\lambda$, then Planck's radiation law is modified. ${ }^{39}$ As discussed in the section on 'Radiation noise', for a body in thermal equilibrium, the emissivity at every wavelength equals the absorption at that wavelength. Hence, artificial materials with a structuredependent emissivity will also have a structure-dependent absorptivity. Hesketh et al. ${ }^{40}$ have demonstrated that the emissivity from deep surface gratings with a period comparable to the wavelength is different from the emissivity of a smooth surface. The emissivity was found to have a dependence on the grating period, which the authors attributed to standing wave modes generated in the deep gratings. More recently, surface gratings in polar materials have been used to generate thermal emission that is spectrally and spatially coherent for transverse magnetic (TM) polarization. ${ }^{6}$ This behavior was attributed to generation of surface phonon polariton modes in the grating. Figure $4 \mathrm{a}$ shows the atomic force microscope (AFM) image of this grating while Figure $4 \mathrm{~b}$ shows the emissivity as a function of polar angle for three different wavelengths: $11.04 \mu \mathrm{m}$ (blue), $11.36 \mu \mathrm{m}$ (red) and $11.86 \mu \mathrm{m}$ (green). It can be seen that strong absorption is obtained over a small angular region and over a narrow spectral region. This strong absorption is obtained by modifying the surface of a reflective material.

Two and three dimensional photonic crystals have also been used to achieve spectrally selective absorption. ${ }^{21,27}$ Fleming et al. ${ }^{27}$ fabricated a three-dimensional photonic crystal in tungsten and achieved a complete photonic bandgap between 8 and $20 \mu \mathrm{m}$. A scanning electron microscope (SEM) image of this photonic crystal is seen in Figure $5 \mathrm{a}$. This photonic bandgap results in strong reflection over a broad range of angles. The photonic crystal structure also showed strong absorption peak at the band edge at $\sim 6 \mu \mathrm{m}$ as shown in Figure $5 \mathrm{~b}$ in red. The peak absorption is enhanced to as much as $50 \%$, which is much higher than a flat tungsten surface. This enhancement in absorption was later shown to be due to slower group velocity at the band edge leading to a longer interaction of the field with intrinsically absorbing metal. ${ }^{41}$

There has been a significant effort to develop spectrally selective absorbers using plasmonics and metamaterials at several different wavelengths. ${ }^{42-44}$ The general architecture of these devices is similar. It consists of a top metallic subwavelength structure that is separated from a highly reflecting back mirror by a thin dielectric. The shape and size of the top metal and the thickness of the dielectric determine the location of absorption resonance. Liu et al. ${ }^{8}$ recently demonstrated the use of such metamaterials for single band and dual band thermal emission from heated structures. Figure 6 shows the narrow band absorptivity obtained in their metamaterial absorbers. The top panel shows the absorptivity for a single size metamaterial, while the bottom panel shows the absorptivity for a metamaterial with two different sized structures, having absorption in two wavelength bands.

Though there has not been a rigorous mathematical treatment of the absorption mechanism, powerful numerical simulation techniques have been used to design these structures and gain some insight into the absorption mechanism. The simulations show that the magnetic field component of the incoming radiation generates antiparallel currents in the top and bottom metal, creating a strong field confinement at resonance. There is a separate resonance peak associated with 


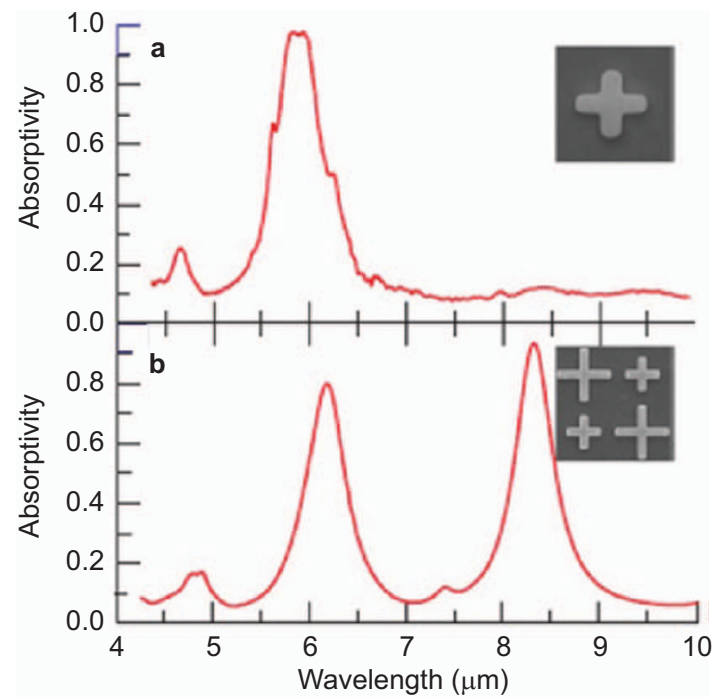

Figure 6 Spectral absorptivity of a metamaterial with (a) single sized feature and (b) two sizes of features from Ref. 8. TM, transverse magnetic; TE, transverse electric.

LC oscillation in the top structure. The absorption is through a combination of ohmic losses in metal and dielectric losses in the spacer. One interesting feature of this type of resonant absorption is that, if the correct mode is excited, the spectral location of the resonance does not shift with the angle of incidence, as would be the case with a simple Fabry-Perot cavity coupled absorber. Figure 7 shows a contour plot of absorptivity $v s$. wavelength and angle for a metamaterial absorber fabricated in gold on a flexible Kapton layer. ${ }^{43}$ The absorber is designed to be dual band, with peak resonance at 3.3 and $3.9 \mu \mathrm{m}$ wavelengths. It can be seen that the peak of the absorption spectrum does not shift much with the angle of incidence for both TE and TM polarizations.

\section{BROADBAND THERMAL DETECTOR TECHNOLOGY}

\section{Flat spectrum}

Two of the easiest ways to achieve a flat spectral response are using metallic films or metal blacks. Detailed theoretical analysis of absorption in metallic films, and of filters constructed from such films, was given by Hadley and Dennison. ${ }^{45}$ They showed that the maximum theoretical absorption of a thin metal film in air is $50 \%$ for a sheet resistance of $189 \Omega /$ square, although this paper and many others assume the good conductor model of metal optics where the magnitudes of the real and imaginary parts of the indices of refraction are equal. This is seldom the case, even approximately, in real metals. Metal black coatings are commonly used to give absorption values higher than specular metal films. ${ }^{46}$ These films can be prepared in a variety of ways including, evaporation in gaseous ambient, galvanic processes, or chemical dipping processes. ${ }^{47,48}$ The performance of metal blacks is good $(>95 \%)$ at shorter wavelengths but starts to deteriorate at longer wavelengths in the infrared. Black paints like Nextel black have good absorption, but their thermal mass is too high to be useful for thermal detectors. ${ }^{48}$ Gold black coatings were evaluated for use in pyroelectric detectors by Lehman, who measured a broadband absorption higher than $90 \%$ from 1 to $10 \mu$ m wavelength range. ${ }^{49}$ Ultra black coatings made from an alloy of nickel and platinum have also been reported for use in optical detectors. ${ }^{50}$ The performance of this alloy was found to be better than gold black in the visible to near infrared range. Silicon-based devices with copperconstantan thermopiles have been developed that, while having a rising response at wavelengths longer than $8 \mu \mathrm{m}$, have a relatively flat response in the $1-8 \mu \mathrm{m}$ range. ${ }^{51}$

In recent years, significant research has been conducted using carbon nanotubes for broadband absorber coatings. A 'forest' of vertically aligned multiwalled carbon nanotubes (CNT) was demonstrated to have extremely high absorption in the visible region. ${ }^{52}$ Blackbody-type high absorption was also demonstrated for vertically aligned single-walled carbon nanotubes over a very broad range of wavelengths, from 0.2 to $200 \mu \mathrm{m} .{ }^{53}$ This effect is due to the sparse structure of the forest and vertical alignment of the CNTs. Incident light is reflected into the bulk of the film and, due to multiple absorptions, very little light escapes out. These coatings were utilized as absorbers for large area pyroelectric detectors ${ }^{54}$ and for reducing stray light in space borne instruments. ${ }^{55,56}$

\section{Antiresonant interference structures}

Antiresonance structures employ a quarter wave gap between a bottom reflector and the detector to couple a broadband signal into the absorbing bolometer. The bottom mirror is usually metallic. This leads to broadband coupling of radiation into the absorbing plate around the center wavelength as any radiation passing through
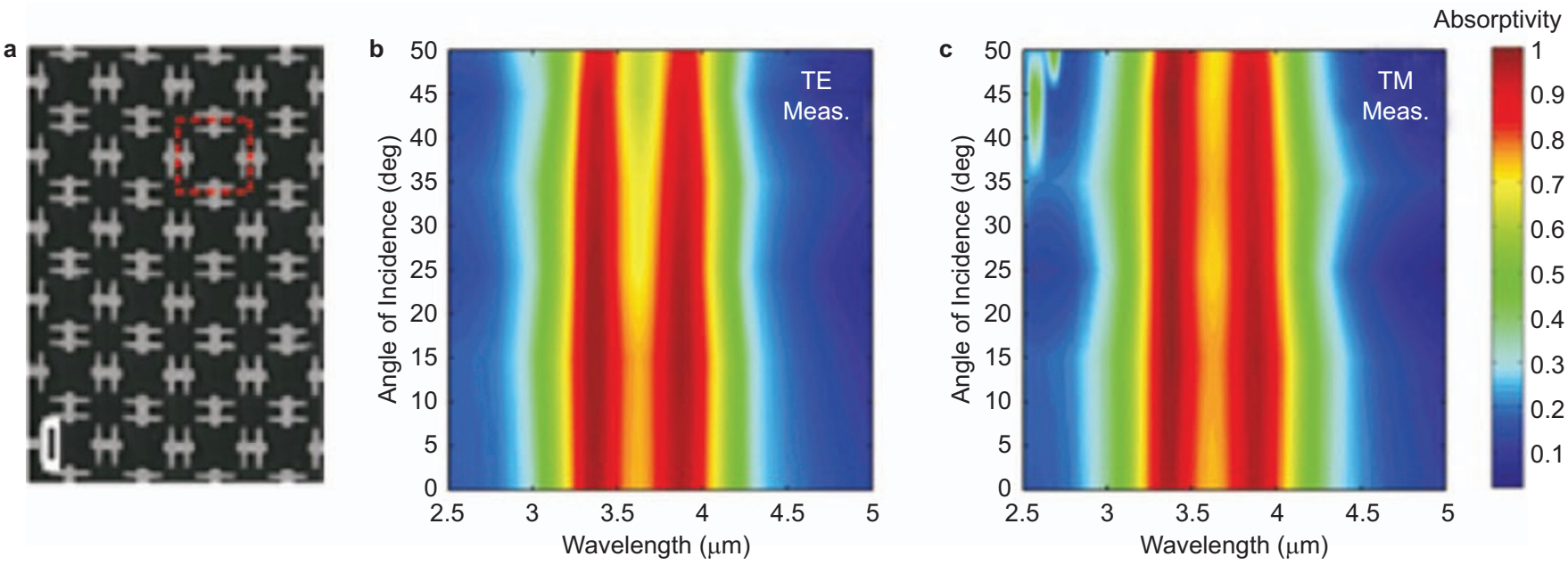

Figure 7 (a) SEM image of metamaterial absorber. Spectral and spatial absorptivity for (b) TE wave and (c) TM wave from Ref. 43. 


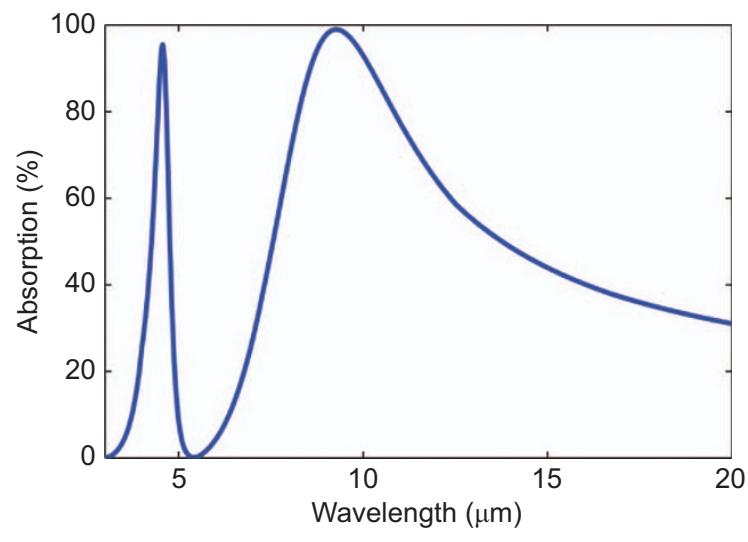

Figure 8 Simulated absorption spectrum of simple antiresonance device using a 10-nm Ti absorber, 160-nm Ge sensing layer, 250-nm air gap, and a perfect reflector showing $>50 \%$ absorption in the $8-14 \mu \mathrm{m}$ range.

the plate is then reflected off the lower surface back to the absorber, which rests at the intensity maximum of the interference pattern. This structure is often called a 'resonant cavity' in the literature, but this is misnomer since it is exactly the opposite: an antiresonant structure with phase characteristics far closer to a distributed Bragg reflector than an optical cavity. A simple simulation of the absorption of such a device can be seen in Figure 8. The device simulated includes a $10-\mathrm{nm}$ $\mathrm{Ti}$ absorber and 160-nm Ge sensing layer suspended over a perfect reflector by a $250-\mathrm{nm}$ air gap. The simulation shows an absorption of greater than $50 \%$ in the $8-14 \mu \mathrm{m}$ range. The simulation is done using the $2 \times 2$ matrix technique from $\mathrm{Yeh}^{20}$ and optical constants from Palik. ${ }^{57}$

Though fabrication techniques for antiresonant thermal detectors vary, most follow the same basic outline. A metallic mirror is deposited and patterned on a substrate which is often pre-patterned with readout circuitry. A sacrificial layer, often polyimide or $\mathrm{SiO}_{2}$, is deposited on the substrate at a thickness corresponding to the desired air gap. The sacrificial layer is then patterned to create vias, which allow for electrical connection and structural support between the substrate and the bolometer device. Next, the bolometer is formed by depositing and patterning the absorber and resistor materials. Often another material is used to protect the absorber material from etchants and provide structural support. Finally, the sacrificial material is removed using a wet or plasma etch leaving the bolometer plate suspended over the substrate.

Early work on antiresonant microbolometers was done at Honeywell and Texas Instruments. ${ }^{58,59}$ The work of Cole details a device with a vanadium oxide absorbing layer, phosphate glass sacrificial layer, silicon nitride support structure, and $1-2 \mu \mathrm{m}$ gap spacing. This work details the effect of gap spacing on the absorption throughout the longwave infrared (LWIR) spectrum and presents a device with absorption over $80 \%$ in the $8-14 \mu \mathrm{m}$ range. The work of Hornbeck at Texas Instruments involves an antiresonant microbolometer fabricated directly onto a CMOS wafer to provide readout circuitry for a $256 \times 256$ focal plane array. ${ }^{59}$ This device consists of a plate containing

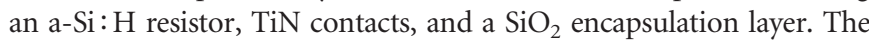
plate is suspended over the substrate using $\mathrm{Ti}: \mathrm{W}$ interconnects and the substrate area below the plate is covered with $\mathrm{Al}$ to enhance reflection, while the gap is formed using a polyimide sacrificial layer.

The modern microbolometer focal plane array market is dominated by microbolometers with antiresonance configurations. Because of this, a majority of research in this field is done by research laboratories

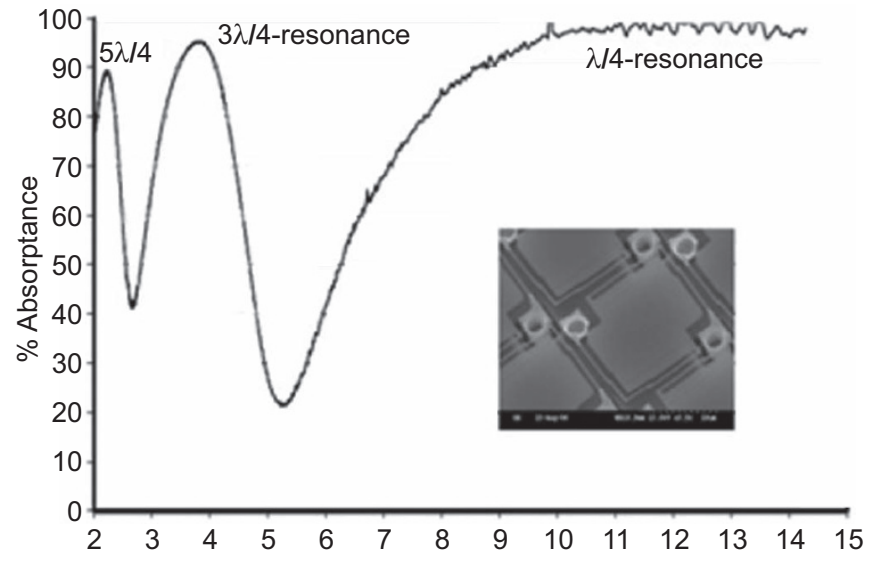

Figure 9 Absorption spectrum of a modern standard design antiresonance microbolometer developed by L-3. An SEM image of the device can be seen in the inset. ${ }^{61}$ SEM, scanning electron microscope.

at companies like Raytheon, ULIS, BAE, L-3, DRS and others. Though the standard configuration is still in use, other configurations like the umbrella design ${ }^{60}$ have been developed to meet industry needs.

An example of modern bolometers using the standard configuration comes from L-3 Communications Infrared Products. ${ }^{61}$ This work uses a-Si and a-SiGe sensor layers due to the favorable temperature coefficient of resistance of these materials. Other design details of the device are withheld, but the optical spectrum created by the gap between the bolometer plate and a metallic reflector can be seen in Figure 9, as well as a SEM image of the device in the inset.

The umbrella configuration of antiresonant microbolometers was developed at DRS Sensors \& Targeting (Cypress, CA, USA). ${ }^{60}$ As opposed to the single layer standard configuration, the umbrella configuration is a two-layer design. In order to increase pixel fill factor the absorbing layer is separated from the readout circuitry. A slightly more complex optical stack is then formed between the absorbing layer, readout layer, and substrate layer. The substrate layer can be coated with a metallic reflector to form a gap with the absorber layer, while the gap spacing between the readout layer and the other two layers can be fabricated to further optimize the spectrum. A schematic and SEM image from DRS can be seen in Figure $10 .{ }^{62}$ DRS uses an Al or Ti reflector, $\mathrm{VO}_{x}$ or $\mathrm{TiO}_{x}$ readout layer, and an undefined dielectric absorber. This configuration leads to a broad absorption with $>50 \%$ relative spectral response from 3 to $17 \mu \mathrm{m} .{ }^{63}$ A similar design to the DRS umbrella configuration was developed around the same time by NEC. ${ }^{64}$ This design uses an umbrella-like structure only at the edges to cover the support structure. This device uses a $\mathrm{SiN}_{x}$ absorber, $\mathrm{VO}_{x}$ sensor layer, and metallic reflecting layer with both gaps defined by two polyimide sacrificial layers. ${ }^{65}$

The modeling of an interesting variation on the standard configuration is the two-color sensor presented by Almasri et al. ${ }^{66}$ This configuration includes a bolometer plate consisting of a NiCr absorber and amorphous $\mathrm{Si}$ resistor suspended over an $\mathrm{Al}$ mirror. The $\mathrm{Al}$ mirror is suspended over the substrate so that the gap between the sensor layer and mirror layer is tunable, allowing for a single sensor to either detect or reject the mid wave infrared (MWIR) while detecting the LWIR. The device can be tuned between a cavity depth of 0.9 and $2.15 \mu \mathrm{m}$ to switch between MWIR absorbing and LWIR absorbing modes as seen in Figure 11.

A method for achieving high performance in the MWIR but not the LWIR is to use materials that absorb only in the desired spectral 

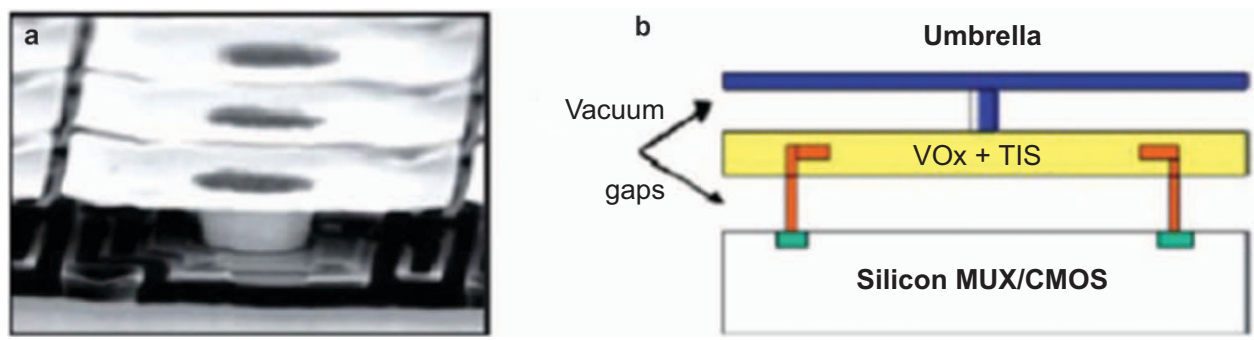

Figure 10 SEM image (a) and schematic (b) of a microbolometer using the 'umbrella design' from DRS Inc. ${ }^{62}$ SEM, scanning electron microscope.

range. One example of this is to use a narrow bandgap semiconductor. The theoretical radiation noise of a device of this sort is shown as a function of cutoff wavelength in Figure 12. This cutoff wavelength is an idealization of a semiconductor, with perfect absorption at wavelengths below the bandgap and perfect transmission at wavelengths above. Shea et al. ${ }^{67}$ model a structure that uses PbSe semiconductor as the absorbing layer in a microbolometer combined with optical interference with a back reflector to enhance absorption in the 3-5 $\mu \mathrm{m}$ wavelength range. The PbSe does not absorb light of wavelength longer than about $5 \mu \mathrm{m}$, so no undesired signals are detected from the LWIR. There are many plasmonic, nanoparticles, and other approaches that could achieve this sort of broadband spectrally selective absorption as well.

\section{SPECTRALLY SELECTIVE DETECTOR TECHNOLOGY}

As the section on 'Spectral properties of infrared materials: artificial' described, there has been a great deal of research work on novel infrared absorbers and emitters in recent years; however, relatively little has been applied to detection. This is one of the primary reasons why we feel that a renaissance of interest in the area of spectrally selective thermal detectors is likely. This being said, there have been some very interesting developments in the field and in this section, we survey some of this work.

In noise theory, there have been a number of works, particularly dealing with quantum optical or cryogenic topics. An example is by Boyd, ${ }^{68}$ who discusses noise fluctuations specific to the long wavelength limit of background radiation, $h v<<k T$, and calculates these for both a broadband device and a cold-filtered and cold-shielded narrowband one. Hanson also presented a theoretical treatment of how reducing the spectral band of a thermal detector could improve its detectivity and proposed that cold shielded systems would be able to achieve this. ${ }^{69}$

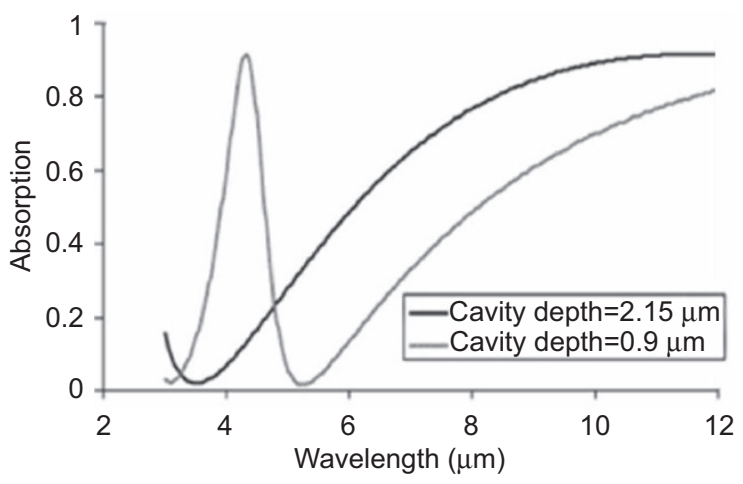

Figure 11 Simulated absorption spectrum of a two-color tunable microbolometer from Ref. 66.
In device-specific work, there have been a variety of approaches to achieving spectral selectivity. One of the oldest is the use of antenna coupling. ${ }^{70,71}$ Historically, this was used to optimally couple radio or terahertz waves into a microbolometer, but with the advent of plasmonics, antenna-based techniques are being used in the infrared as well. A recent infrared antenna-coupled device has been experimentally demonstrated by Gritz et al. ${ }^{72}$ with a small tuning range around $\lambda=10 \mu \mathrm{m}$. Occasionally, it can be somewhat difficult to draw a firm line between antenna-coupled and plasmonic approaches, and indeed, some of the early work using patterned metals might have been called 'plasmonic' had the term existed at the time. More recently, Neikirk's group at the University of Texas modeled a detector plate with a metal grid placed over an air gap and back mirror to enhance absorption in a broad spectral region while rejecting light outside the desired range. ${ }^{73}$ The selectivity of this approach is obtained from the periodicity of the metal pattern and once the pattern is made, the wavelength range is fixed. Experimentally, Maier and Bruckl have shown that infrared absorption in a microbolometer device can be enhanced in a fixed spectral range with a periodically patterned metal-dielectric metal stack. $^{74}$ The absorption characteristics of similar devices were further simulated in Ref. 75. The devices were discussed as metamaterials, but data for the dielectric permittivity and magnetic permeability were not presented. There has been recent work by Renoux et al. ${ }^{76}$ at the University of Iceland on subwavelength platinum wires as bolometers. While these were not meant to be

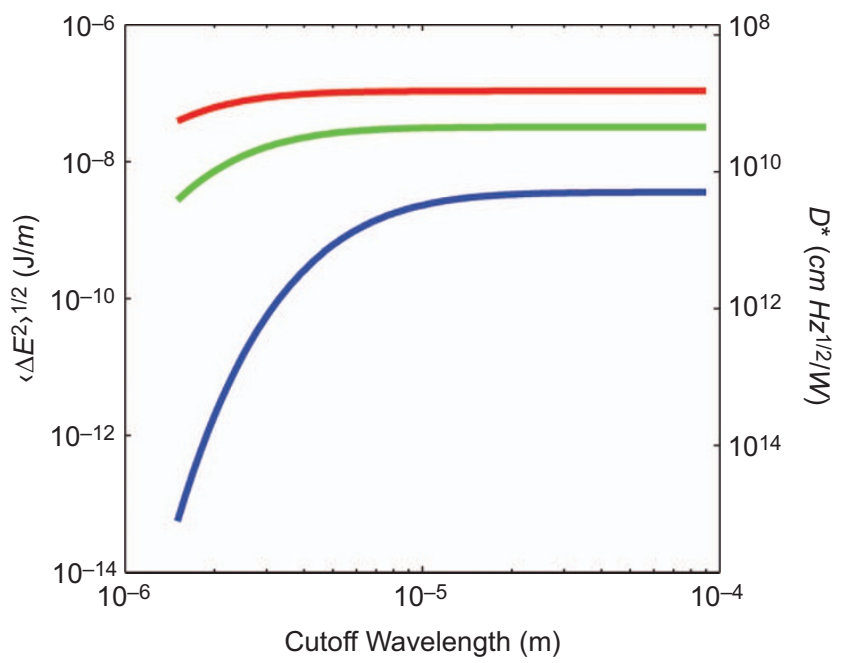

Figure 12 RMS energy fluctuations (left) and detectivity (right) vs. cutoff wavelength for an ideal semiconductor absorber $\left(\varepsilon=1\right.$ for $\lambda<\lambda_{\text {cutoff }}$ and 0 elsewhere). The detector is at room temperature $(290 \mathrm{~K})$ while the background is plotted at $290 \mathrm{~K}$ (blue), $800 \mathrm{~K}$ (green) and $1300 \mathrm{~K}$ (red). RMS, root mean square. 


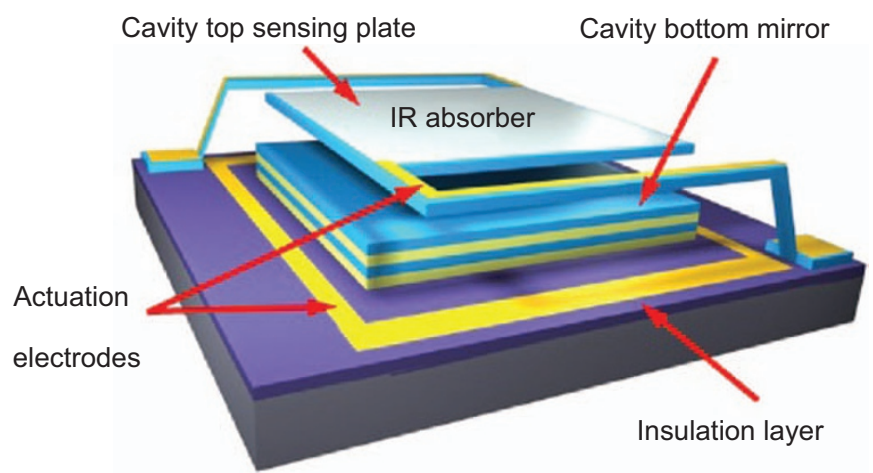

Figure 13 Conceptual diagram of a tunable absorption filter for LWIR microbolometers. The device can continuously tune the absorption resonance and switch between two finesse states. The IR absorber is deposited on the top of the upper plate. The bottom DBR mirror has reflectivity centered at $10 \mu \mathrm{m}$. The primary actuation electrodes are on the support beams and substrate to delay snap-through and enhance tuning range. ${ }^{24}$ LWIR, long-wave infrared; DBR, distributed bragg reflector.

spectrally selective devices, one could take advantage of interference effects to create an array or other pattern of such wires to achieve spectral selectivity.

Our own research group's interest in thermal detectors began in early 2000 with an investigation of coupled-absorption microbolometers, and this work led to a project under DARPA's Adaptive Focal Plane Program. Results and an image from the devices that were eventually constructed are shown in Figures $13-15 .^{24,77}$ The design utilizes a coupled-absorption cavity as described in the noise theory section, but the absorber is part of the top mirror rather than a separate structure. The microbolometer filters used electrostatic actuation between the top and bottom mirrors to control the air gap spacing. The devices were continuously tunable from $\lambda=8.7 \mu \mathrm{m}$ to $\lambda=11.1 \mu \mathrm{m}$, with a broader band mode at higher actuation voltage at approximately $\lambda=11.3 \mu \mathrm{m}$. Razansky et al. ${ }^{78}$ has modeled a similar structure as a plasmonic resonance device.

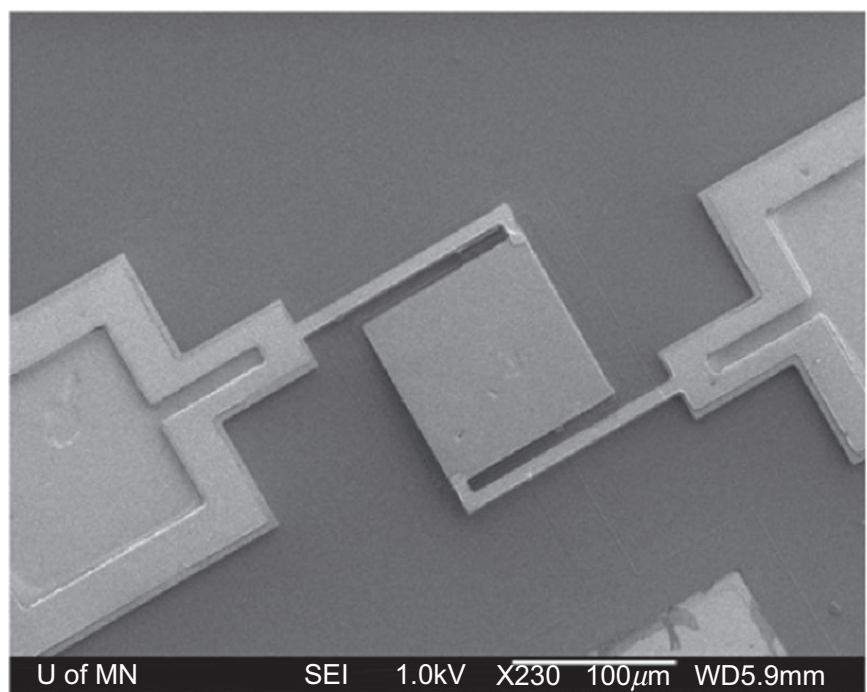

Figure 14 SEM image of a device after etch release. The top plate is $100 \mu \mathrm{m} \times 100 \mu \mathrm{m}$ and is supported by two $150 \mu \mathrm{m} \times 5 \mu \mathrm{m}$ beams. Chromium/ gold has been evaporated on the beams and anchors for actuation. The two structures to the left and right are bonding pads for later testing. ${ }^{77}$ SEM, scanning electron microscope.

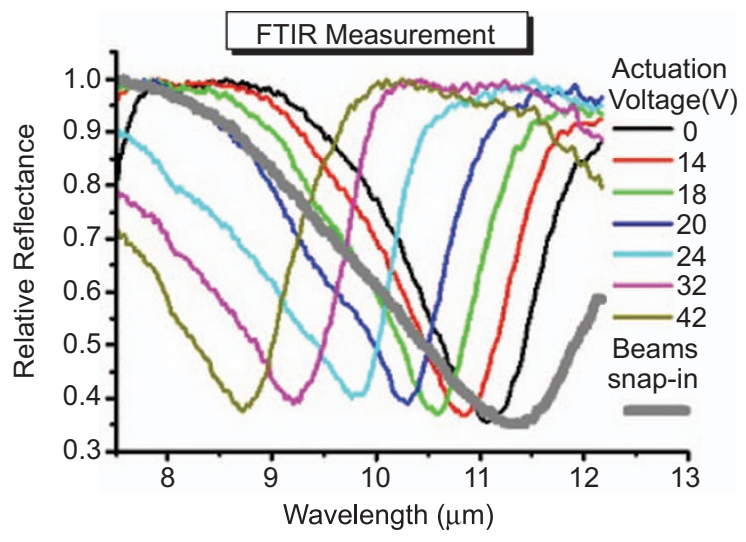

Figure 15 FTIR measurement of the relative reflectance spectra of a $120 \mu \mathrm{m} \times 120 \mu \mathrm{m}$ size filter. As the applied voltage is varied from 0 to $42 \mathrm{~V}$, the narrowband resonance shifts from 11.1 to $8.7 \mu \mathrm{m}$. A broadband resonance centered at $11.3 \mu \mathrm{m}$ is created when the voltage is increased to $45 \mathrm{~V} .{ }^{24} \mathrm{FTIR}$, Fourier transform infrared spectroscopy.

The use of infrared materials to achieve spectrally selective infrared absorption is an active research area as described in sections on 'Spectral properties of infrared materials: bulk' and on 'Spectral properties of infrared materials: artificial', but few have been formally incorporated into thermal detectors. Preliminary work from Gawarikar et al. ${ }^{20,79}$ has measured the spectral characteristics, thermal conductance, and detectivity of microbolometers with $\mathrm{SiO}_{2}$ absorbing films. $\mathrm{SiO}_{2}$ has an absorption peak between 9.0 and $10 \mu \mathrm{m}$ and the devices absorbed strongly in this range despite their small thickness. These devices had thermal conductance that was radiation-limited and well below the blackbody value for broadband absorbers.

\section{SPECULATIONS ON THE FUTURE}

Guessing what the next decade or two will bring for thermal detector technology is difficult, but there are several developments that can provide us with a good starting point. The first is the tremendous advance of standard broadband imaging arrays. Uncooled infrared microbolometer cameras are cheaper and more readily available than ever before, but most of the technology development has gone into fabricating cameras that put pixel density and array size above sensitivity, making great images rather than great sensors. For some systems, such as high-end military units, this will continue. The pixel sizes of microbolometers are approaching the wavelength of thermal light at room temperature $(\lambda \approx 10 \mu \mathrm{m})$, but only the most foolhardy would predict that pixel scaling will stop at this 'limit,' especially given how optical advances overcame the dire predictions about optical lithography for very large scale integration (VLSI) a few decades ago. However, there will be many systems, such as chemical sensing, where multimegapixel images are not necessary and technology development will shift towards sensitivity and spectral characteristics.

Many spectral characteristics have been reviewed here, but very narrow spectral bands in thermal detectors do not seem to have been heavily pursued to date. One reason for this may be that devices have been designed to assist military reconnaissance and target recognition, where many spectral bands are not necessary. Exceptions that are narrowband are usually the trivial cases of placing a Fabry-Perot filter or grating monochromator in tandem with an otherwise standard detector, but these do not take advantage of the noise improvements one can obtain by limiting the spectrum. Research into nanoparticles and patterned materials, especially metals, 
metamaterials, and photonic crystals will continue, and devices with these will be made with narrower and narrower bands. Experiments with these materials will trigger a new look at some of the optical constants of films used in the infrared. Most modeling in the infrared is done with optical constants developed for thick materials, but plasmonics will drive new studies to measure the constants of films of a few atomic thicknesses. For example in Ref. 24, the Cr layer used to assist reflection and absorption is $1.8 \mathrm{~nm}$ thick. The use of doped semiconductors as tunable infrared materials will also expand, since the imaginary part of the index of refraction can be altered almost independently of the real part over a wide doping range, and the plasma frequency can be shifted through much of the infrared.

The sensitivity of thermal detectors at room temperature has a great deal of room to improve. The best detectivities that have been reported at room temperature are in the range of a few times $10^{9} \mathrm{~cm} \mathrm{~Hz}^{1 / 2} \mathrm{~W}^{-1}$. An example using micro- and nanowires is described in Ref. 76 . The fundamental radiation limit of a broadband thermal detector is almost an order of magnitude higher than this and spectrally selective detectors even better still, as seen in Figures 1 and 3. To achieve these higher detectivities, the highly advanced thermal isolation structure of current microbolometer arrays will be adapted for somewhat larger pixels (for reasons of both sensitivity and spectral selectivity), and we believe, that thermoelectric transducers will be preferred over bolometric to overcome $1 / f$ noise at the highest sensitivities.

If narrowband thermal detectors are made tunable and operated at close to their radiation limit, then they will find uses in gas and chemical detection of common gases. One of the biggest advantages of Fourier transform infrared (FTIR) spectrometers is the multiplex or Fellgett advantage that they enjoy over monochromators and FabryPerot filters. This advantage arises, because the FTIR spectrometer collects signal and noise from all wavelength bands at once. Current monochromators-plus-detectors and filters-plus-detectors receive signal from only one waveband while receiving noise from all wavelengths, because their detectors are broadband. If one has a background-limited tunable detector, such as a coupled-absorption cavity, where both the signal and noise are spectrally selective, then the multiplex advantage disappears. The FTIR has other advantages for complex chemicals and systems with many chemicals, but for simple gases and gas combinations, spectrally selective thermal detectors will provide a more compact and inexpensive alternative.

\section{ACKNOWLEDGMENTS}

The authors would like to acknowledge funding for the work described herein from DARPA, ARO, the Electro-Optics Center, and the University of Minnesota, particularly DARPA/ARO contract W911QX-12-C-0002. We would also like to thank the authors and publishers who kindly gave permission to their figures. Finally, we would like to thank Nibir Dhar, John Carrano, Ray Balcerak, Bill Clark, Joe Pelligrino and Andrew Wood for much useful advice over the years.

1 Schwab K, Henriksen EA, Worlock JM, Roukes ML. Measurement of the quantum of thermal conductance. Nature 2000; 404: 974-977.

2 Roukes ML. Yoctocalorimetry: phonon counting in nanostructures. Physica B: Condens Matt 1999; 263-264: 1-15.

3 Cahill DG, Ford WK, Goodson KE, Mahan GD, Majumdar A et al. Nanoscale thermal transport. J Appl Phys 2003; 93: 793-818.

4 Gabrielson TB. Mechanical-thermal noise in micromachined acoustic and vibrationsensors. IEEE Trans Electron Dev 1993; 40: 903-909.

5 Cleland AN, Roukes ML. Noise processes in nanomechanical resonators. J Appl Phys 2002; 92: 2758-2769.

6 Greffet JJ, Carminati R, Joulain K, Mulet JP, Mainguy S et al. Coherent emission of light by thermal sources. Nature 2002; 416: 61-64.
7 Pralle MU, Moelders N, McNeal MP, Puscasu I, Greenwald AC et al. Photonic crystal enhanced narrow-band infrared emitters. Appl Phys Lett 2002; 81: 4685-4687.

8 Liu X, Tyler T, Starr T, Starr AF, Jokerst NM et al. Taming the blackbody with infrared metamaterials as selective thermal emitters. Phys Rev Lett 2011; 107: 045901.

9 Jones RC. The ultimate sensitivity of radiation detectors. J Opt Soc Am 1947; 37: 879-888.

10 Lewis WB. Fluctuations in streams of thermal radiation. Proc Phys Soc 1947; 59: 34-40.

11 Wormser EM. Properties of thermistor infrared detectors. J Opt Soc Am 1953; 43 : 15-19.

12 Golay MJE. The theoretical and practical sensitivity of the pneumatic infrared detector. Rev Sci Instrum 1949; 20: 816-820.

13 Ruck GT, Barrick DE, Stuart WD, Krichbaum CK. Radar Cross Section Handbook, Vol. 2. New York: Plenum; 1970.

14 Knott E, Shaeffer JF, Tuley MT. Radar Cross Section, 2nd ed. Raleigh: Scitech; 2004.

15 Emerson WH. Electromagnetic wave absorbers and anechoic chambers through the years, IEEE Trans Antennas Propag 1973; AP-21: 484-490

16 Fano RM. Theoretical limitations on the broadband matching of arbitary impedances II. J Franklin Inst 1950; 249: 139-154.

17 Rozanov KN. Ultimate thickness to bandwidth ratio of radar absorbers. IEEE Trans Antennas Propag 2000; 48: 1230-1234.

18 Fellgett PB. On the ultimate sensitivity and practical performance of radiation detectors. J Opt Soc Am 1949; 39: 970-976.

19 Fox M. Quantum Optics: An Introduction. Oxford: Oxford University Press; 2006.

20 Gawarikar AS, Shea RP, Mehdaoui A, Talghader JJ. Radiation heat transfer dominated microbolometers. Proceedings 2008 IEEE/LEOS International Conference on Optical MEMs and Nanophotonics; IEEE, 2008, pp178-179.

21 Greffet JJ, Carminati R, Joulain K, Mulet JP, Mainguy S et al. Coherent emission of light by thermal sources. Nature 2002; 416: 61-64.

22 Pralle MU, Moelders N, McNeal MP, Puscasu I, Greenwald AC et al. Photonic crystal enhanced narrow-band infrared emitters. Appl Phys Lett 2002; 81: 4685-4687.

23 Bean JC, Qi JM, Schow CL, Li R, Nie H et al. High-speed polysilicon resonant-cavity photodiode with $\mathrm{SiO}_{2}-\mathrm{Si}$ Bragg reflectors. IEEE Photon Technol Lett 1997; 9: 806-808.

24 Wang Y, Potter BJ, Talghader JJ. Coupled absorption filters for thermal detectors. Opt Lett. 2006; 31: 1945-1947.

25 Wan W, Chong Y, Ge L, Noh H, Stone AD et al. Time-reversed lasing and interferometric control of absorption. Science 2011; 331: 889-892.

26 Yeh P. Optical Waves in Layered Media. New York: Wiley; 1988.

27 Fleming JG, Lin SY, El-Kady I, Biswas R, Ho KM. All-metallic three-dimensional photonic crystals with a large infrared bandgap. Nature 2002; 417: 52-55.

28 Jackson JD. Classical Electrodynamics. 3rd ed. New York: Wiley; 1998.

29 Kittel C. Introduction to Solid State Physics. 8th ed. New York: Wiley; 2004.

30 Kittel C, Kroemer H. Thermal Physics. 2nd ed. New York: W. H. Freeman; 1980.

31 Houghton JT. Infra-red Physics. Oxford: Clarendon Press; 1966.

32 Fox M. Optical Properties of Solids. 2nd ed. Oxford: Oxford University Press; 2010.

33 Kyle TG. Atmospheric Transmission, Emission and Scattering. 1st ed. Oxford: Pergamon; 1991.

34 Briggs HB, Fletcher RC. Absorption of infrared light by free carriers in germanium. Phys Rev 1953; 91: 1342-1346.

35 Haas C. Infrared absorption in heavily doped n-type germanium. Phys Rev 1962; 125: 1965-1971.

36 Pankove JI, Aigrain P. Optical absorption of arsenic-doped degenerate germanium. Phys Rev 1962; 126: 956-962.

37 Spitzer WG, Trumbore FA, Logan RA. Properties of heavily doped n-type germanium. J App/ Phys 1961; 32: 1822-1830.

38 Spitzer WG, Fan HY. Determination of optical constants and carrier effective mass of semiconductors. Phys Rev 1957; 106: 882-890.

39 Baltes HP. Planck's radiation law for finite cavities and related problems. Infrared Phys 1976; 16: 1-8.

40 Hesketh PJ, Zemel JN, Gebhart B. Organ pipe radiant modes of periodic micromachined silicon surfaces. Nature 1986; 324: 549-551.

41 Lin SY, Fleming JG, Li ZY, El-Kady I, Biswas R et al. Origin of absorption enhancement in a tungsten, three-dimensional photonic crystal. J Opt Soc Am B 2003; 20: 1538-1541.

42 Liu X, Starr T, Starr AF, Padilla WJ. Infrared spatial and frequency selective metamaterial with near-unity absorbance. Phys Rev Lett 2010; 104: 207403.

43 Jiang ZH, Yun S, Toor F, Werner DH, Mayer TS. Conformal dual-band nearperfectly absorbing mid-infrared metamaterial coating. ACS Nano 2011; 5: 4641-4647.

44 Liu N, Mesch M, Weiss T, Hentschel M, Giessen H. Infrared perfect absorber and its application as plasmonic sensor. Nano Lett 2010; 10: 2342-2348.

45 Hadley LN, Dennison DM. Reflection and transmission interference filters. J Opt Soc Am 1947: 37: 451-453.

46 Harris L. The transmittance and reflectance of gold black deposits in the 15- to 100 micron region. J Opt Soc Am 1961; 51: 80-82.

47 Lang W, Kühl K, Sandmaier H. Absorbing layers for thermal infrared detectors. Sens Actuators A 1992; 34: 243-248.

48 Betts DB, Clarke FJJ, Cox LJ, Larkin JA. Infrared reflection properties of five types of black coating for radiometric detectors. J Phys E 1985; 18: 689-696.

49 Lehman J, Theocharous E, Eppeldauer G, Pannell C. Gold-black coatings for freestanding pyroelectric detectors. Meas Sci Technol 2003; 14: 916-922. 
50 Kodama S, Horiuchi M, Kunii T, Kuroda K. Ultra-black nickel-phosphorus alloy optical absorber. IEEE Trans Instrum Meas 1990; 39: 230-232.

51 Srinivas TAS, Timans PJ, Butcher RJ, Ahmed H. 'A free-standing microthermopile detector,' Sens Actuators A 1992, 32, 403-406.

52 Yang ZP, Ci L, Bur JA, Lin SY, Ajayan PM. Experimental observation of an extremely dark material made by a low-density nanotube array. Nano Lett 2008; 8: 446-451.

53 Mizuno K, Ishii J, Kishida H, Hayamizu Y, Yasuda S et al. A black body absorber from vertically aligned single-walled carbon nanotubes. Proc Natl Acad Sci 2009; 106: 6044-6047.

54 Lehman J, Engtrakul C, Gennett T, Dillon A. Single-wall carbon nanotube coating on a pyroelectric detector. Appl Opt 2005; 44: 483-488.

55 Hagopian JG, Getty SA, Quijada M, Tveekrem J, Shiri R et al. Multiwalled carbon nanotubes for stray light suppression in space flight instruments. Proc SPIE 2010; 7761: 77610F-1-77610F-10

56 Quijada MA, Hagopian JG, Getty S, Kinzer Jr RE, Wollack EJ. Hemispherical reflectance and emittance properties of carbon nanotubes coatings at infrared wavelengths. Proc SPIE 2011; 8150: 815002-1-815002-11.

57 Edward Palik. Handbook of optical constants of solids. San Diego: Elsevier; 1998.

58 Cole BE. Microstructure design for high IR sensitivity. US Patent 5,286,976; 1994

59 Hornbeck LJ. Infrared detector. US Patent 5,021,663; 1991.

60 Skidmore GD, Howard CG. Pixel structure having an umbrella type absorber with one or more recesses or channels sized to increase radiation absorption. US Patent 7,622,717; 2009.

61 Schimert T, Brady J, Fagan T, Taylor M, McCardel W et al. Amorphous silicon based large format uncooled FPA microbolometer technology. Proc SPIE 2008; 6940: 694023-1-694023-7.

62 Li C, Han CJ, Skidmore G. Overview of DRS uncooled VOx infrared detector development. Opt Eng 2011; 50: 061017-1-061017-7.

63 Li C, Skidmore GD, Howard C, Han CJ, Wood L et al. Recent development of ultra small pixel uncooled focal plane arrays at DRS. Proc SPIE 2007; 6542: 65421Y-1$65421 Y-12$.

64 Tohyama S, Kurashina S. Thermal-type infra-red ray solid-state image sensor and method of fabricating the same. US Patent 7,276,698; 2007.

65 Tohyama S, Miyoshi M, Kurashina S, Ito N, Sasaki T et al. New thermally isolated pixel structure for high-resolution $(640 \times 480)$ uncooled infrared focal plane arrays. Opt Eng 2006; 45: 014001-1-014001-10.

66 Almasri M, Bai Xu, Castracane J. Amorphous silicon two-color microbolometer for uncooled IR detection. IEEE Sens J 2006; 6: 293-300.

67 Shea RP, Gawarikar AS, Talghader JJ. Midwave thermal infrared detection using semiconductor selective absorption. Opt Express 2010; 18: 22833-22841.
68 Boyd R. Photon bunching and the photon-noise-limited performance of infrared detectors. Infrared Phys 1982; 22: 157-162.

69 Hanson CM. Uncooled IR detector performance limits and barriers. Proc SPIE 2000 4028: 2-11.

70 Fumeaux C, Gritz MA, Codreanu I, Schaich WL, González FJ et al. Measurement of the resonant lengths of infrared dipole antennas. Infrared Phys Technol 2000; 41. 271-281.

71 Schwarz SE, Ulrich BT. Antenna—coupled infrared detectors. J Appl Phys 1977; 48 1870-1873.

72 Gritz MA, Metzler M, Malocha D, Abdel-Rahman M, Monacelli B et al. Wavelength tuning of an antenna-coupled infrared microbolometer. J Vac Sci Technol B2004; 22 3133.

73 Han SW, Kim JW, Sohn YS, Neikirk DP. Design of infrared wavelength-selective microbolometers using planar multimode detectors. Electron Lett 2004; 40: 1410-1411.

74 Maier T, Brueckl H. Wavelength-tunable microbolometers with metamaterial absorbers. Opt Lett 2009; 34: 3012-3014.

75 Maier T, Brueckl H. Multispectral microbolometers for the midinfrared. Opt Let 2010; 35: 3766-3768.

76 Renoux P, Jonsson S, Klein LJ, Hamann HF, Ingvarsson S. Sub-wavelength bolometers: uncooled platinum wires as infrared sensors. Opt Express 2011; 19 8721-8727.

77 Wang Y, Potter BJ, Sutton M, Talghader JJ. Absorption filters for wavelength tuning and finesse switching of long wave infrared thermal detectors. Proceedings of the Solid State Sensors and Actuators Workshop; June 2006; Transducer Research Foundation: Hilton Head, SC, USA; 260-263.

78 Razansky D, Einziger PD, Adam DR, Tamir T. Optimized microbolometers with higher sensitivity for visible and infrared imaging. Opt Express 2006; 14: 10426-10434.

79 Gawarikar AS, Shea RP, Talghader JJ. Uncooled band-limited microbolometers using $\mathrm{SiO}_{2}$ absorption. Proceedings of the 5th Asia-Pacific Conference on Transducers and Micro-Nano Technology (APCOT); July 2010; Perth, Australia, OMN1, pp164.

This work is licensed under a Creative Commons Attribution-NonCommercial-Share Alike 3.0 Unported License. To view a copy of this license, visit http://creativecommons. org/licenses/by-nc-sa/3.0 